\title{
A BIBLIOTECA MUNICIPAL ORLANDO RIBEIRO E A HOMENAGEM AO SEU TITULAR*
}

SUZANNE DAVEAU ${ }^{1}$

A nova Biblioteca Municipal Orlando Ribeiro (fig. 1) foi inaugurada em Dezembro de 2003 no bairro lisboeta de Telheiras, que se encontrava, na altura, em plena remodelação e desenvolvimento. Os quatro anos decorridos desde então mostram o imediato e durável sucesso desta iniciativa. Situada a dois passos das

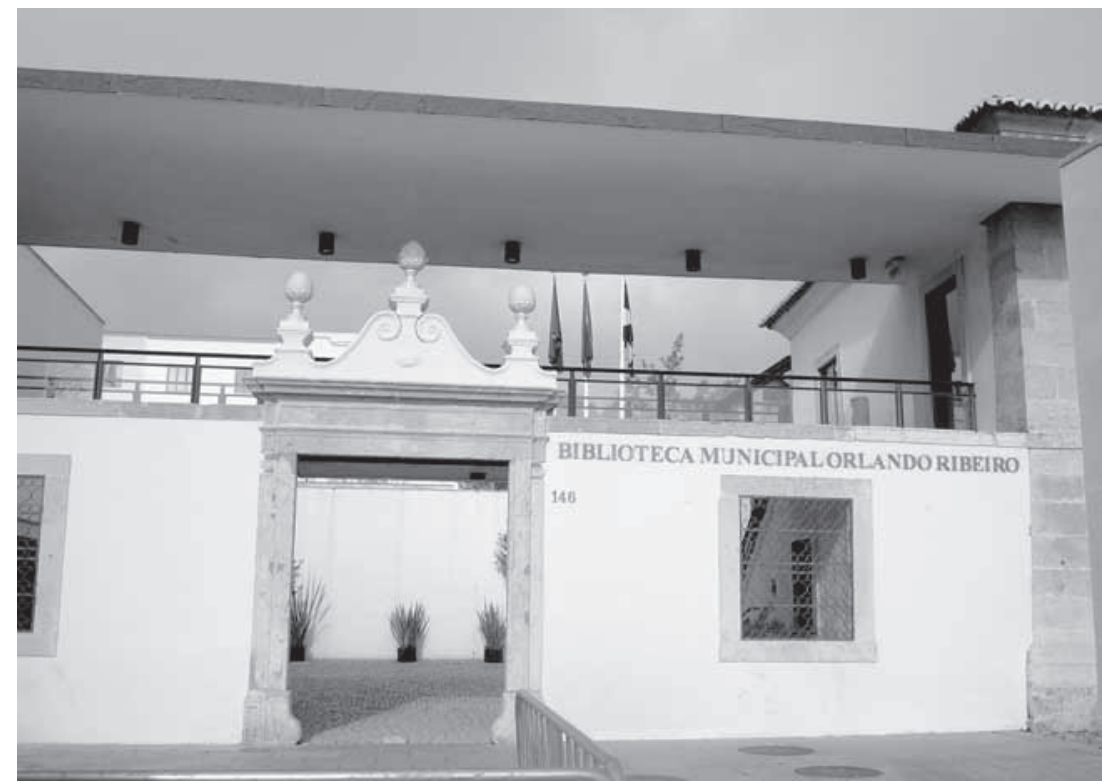

Fig. 1 - A entrada da Biblioteca Municipal Orlando Ribeiro, em Telheiras, Lisboa. Fig. 1 - The entrance of Orlando Ribeiro's city library

* Recebido: 08/10/2007. Revisto: 23/01/2008. Aceite: 27/02/2008.

1 Investigadora do Centro de Estudos Geográficos da Universidade de Lisboa.

E-mail: sdaveau@clix.pt 
artérias mais animadas do novo bairro e da estação de metropolitano que o liga rapidamente às áreas centrais da Lisboa tradicional, a Biblioteca foi inserida de modo feliz na parte preservada do velho caminho que servia de eixo à antiga freguesia. Ergue-se ao lado do Convento das Portas do Céu, núcleo a partir do qual se desenvolveu a aldeia, e da Quinta de São Vicente, velha casa senhorial, há muito transformada em restaurante de recreio, que os colaboradores do Centro de Estudos Geográficos gostavam de frequentar desde que ele ficou instalado, em 1960, na próxima Cidade Universitária (fig. 2).

A Biblioteca ocupa uma parte do oitocentista Solar da Nora, conservando simbolicamente o vistoso engenho metálico para elevação de água que lhe deu o nome. O Arquitecto Duarte Nuno Simões soube habilmente ligar, através do acolhedor pátio de entrada, um velho edifício, recortado em numerosas divisões de dimensão humana onde se abrigam os diversos serviços destinados aos adultos, com o novo edifício, que incorpora um vasto auditório e as salas destinadas aos mais pequenos.

Tranquila, acolhedora, de porta sempre aberta, a Biblioteca conheceu um imediato e sustentado sucesso, que muito teria agradado ao homenageado. Orlando Ribeiro foi, com efeito, desde a infância, grande frequentador das bibliotecas públicas ao seu alcance, tanto no jardim da Praça do Príncipe Real, no bairro lisboeta de São Mamede, como em Viseu durante as férias. Difícil teria sido escolher um tipo de homenagem mais adaptado à sua personalidade e à sua acção cultural. A Biblioteca Municipal Orlando Ribeiro oferece com efeito, em livre acesso, a um público muito diversificado, não apenas livros, mas música, filmes, jornais diários e revistas, terminais informáticos, exposições temporárias e espectáculos diversos. O feliz projecto de um lugar de trabalho e de lazer especialmente dedicado às Viagens pela informação e pelo conhecimento e que fornece aos frequentadores a necessária ajuda para "explorar, investigar, sonhar, descobrir e recriar", deve-se tanto ao brilhante discípulo e amigo próximo, o Prof. Jorge Gaspar, como ao então Presidente da Câmara Municipal de Lisboa, Dr. João Soares, que muito se empenhou no projecto. Deve-se também ao entusiasmo dos primeiros organizadores, os Drs. Álvaro de Matos e Rosa Barreto.

O projecto inicial de homenagem era ambicioso: duas exposições e um ciclo de colóquios sobre as múltiplas facetas da vida e da obra de Orlando Ribeiro. Sem esgotar os possíveis temas, a lista proposta em Outubro de 2003 era aliciante, de modo que aderi com entusiasmo quando me foi proposto tomar conta da sua realização:

\author{
Orlando Ribeiro, Geógrafo \\ Orlando Ribeiro, Encontros e Desencontros com a Política \\ Orlando Ribeiro, Historiador \\ Orlando Ribeiro, Da Fotografia ao Documentário \\ Orlando Ribeiro, Encontros com a Antropologia \\ Orlando Ribeiro, Da Terra \\ Orlando Ribeiro, Percursos Biográficos.
}




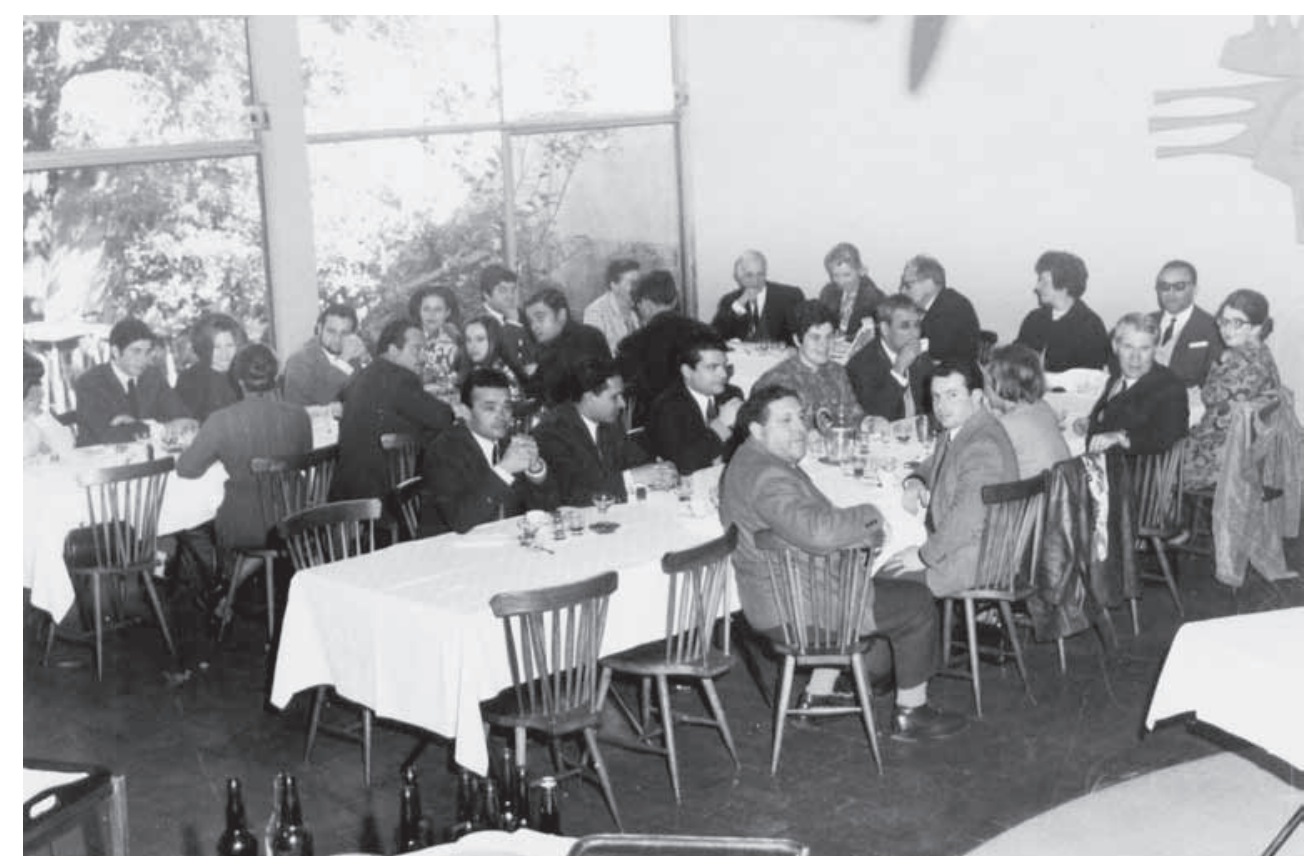

Fig. 2 - Os colaboradores do Centro de Estudos Geográficos festejando, na Quinta de S. Vicente, no dia de anos de Orlando Ribeiro, em 1970.

Fig. 2 - Orlando Ribeiro's birthday commemoration

Ainda se podia pensar noutros temas, como as ligações de Orlando Ribeiro com Espanha, Brasil ou África, ou as suas preocupações sobre religião e organização social. Foram além disso projectados, em Outubro de 2004, novos ciclos de conferências, a organizar pelo Centro de Estudos Geográficos da Universidade de Lisboa, como "Telheiras, um bairro na cidade" ou "Emigração/Imigração".

$\mathrm{Na}$ realidade, os projectos iniciais teriam de ser progressivamente adaptados e reduzidos. A primeira exposição, dirigida em particular ao público mais jovem, dos 4 aos 12 anos, decorreu com grande sucesso, aquando da inauguração da Biblioteca em Dezembro de 2003. Oito jovens desenhadores portugueses encarregaram-se de "recriar" gráfica e imaginariamente oito das viagens de Orlando Ribeiro através do mundo. Interessantes "cadernos de actividades" foram distribuídos aos jovens visitantes da exposição Não se faz bem Geografia quando se faz só Geografia, organizada por Alice Geirinhas e André Ruivo, exposição cheia de vida e de criatividade.

No ano seguinte foram sucessivamente apresentadas duas excelentes exposições fotográficas: Além Mar - Olhares de Orlando Ribeiro, organizada pelo geógrafo Mário Neves, e Orlando Ribeiro, uma Casa no Mundo, do fotógrafo Duarte Belo, que já tinha dedicado ao geógrafo, em 1999, um fascinante livro da Assírio \& Alvim, recheado de fotografias evocativas da sua vida e da sua 
obra. Ao catálogo desta última exposição, com textos de Duarte Belo, Pedro Castro Neves e Rita Neves, o responsável escolheu dar a apresentação dos cadernos de campo tradicionalmente usados pelo homenageado.

Entretanto, decorreram os primeiros colóquios. Mas o sucesso das actividades culturais promovidas pela Biblioteca Municipal Orlando Ribeiro fez com que se tornasse cada vez mais difícil dispor do auditório, normalmente retido com muita antecedência durante as tardes de fim-de-semana. Verificou-se também que a modalidade de colóquios evocativos de uma personalidade já afastada da actualidade, atraía, afinal, pouca gente e, em especial, os mais jovens, que se pretendia interessar com prioridade.

Decidiu-se, primeiro reduzir os colóquios a dois por ano e, depois, realizar apenas os que tinham sido acordados desde o princípio. De tal modo que nem se chegaram a contactar, com grande pena da organizadora, diversas personalidades de relevo, intelectual e afectivamente muito próximas de Orlando Ribeiro. Podiam ter trazido originais testemunhos de grande interesse, sobre as modalidades de influência que a sua obra teve em diversas componentes da sociedade portuguesa. Resta esperar que a larga difusão que se quer dar ao seu Espólio científico suscite, de futuro, novas e interessantes intervenções esclarecedoras.

Foram, finalmente, seis os colóquios realizados em Telheiras, além do que foi consagrado ao tratamento então em curso do Espólio científico, no quadro do Colóquio Silva Telles. Cem Anos de Ensino Superior da Geografia em Portugal (1904-2004), organizado pela Associação Portuguesa de Geógrafos e a Sociedade de Geografia de Lisboa ${ }^{2}$. Ainda que o âmbito da evocação da personalidade orlandeana tenha sido assim arbitrariamente reduzido, os textos que os autores dos colóquios tiveram a gentileza de rever e de completar, com toda liberdade, para a presente publicação, constituem um contributo valioso para a compreensão de uma importante personalidade portuguesa do século XX. Alguns destes autores continuam a aprofundar o tema que tinham apresentado, tendo em preparação estudos mais desenvolvidos. A publicação na revista Finisterra, hoje difundida no país e no mundo não apenas na tradicional forma impressa, mas também através do Sítio Internet do Centro de Estudos Geográficos de Lisboa (http://www.ceg.ul.pt), permitirá tocar um diversificado público, de modo, ao mesmo tempo, aberto e sustentado. Apresentam-se a seguir os seis colóquios, pela ordem de realização.

O primeiro colóquio foi concebido como uma introdução às sessões seguintes e incidiu, portanto, fundamentalmente sobre os anos de formação do geógrafo. A sua infância e o seu ambiente familiar foram apresentados por Suzanne Daveau, através de uma selecção de fotografias ilustrando a sua dupla origem, lisboeta e beirã. Oriundo de um estrato social popular, Orlando Ribeiro teve no entanto, desde novo, ocasiões de estabelecer relações com personalidades de alta categoria intelectual, por ter a sorte de viver num bairro universitário. Entretanto,

2 Este Colóquio será lembrado na terceira parte do presente número da Finisterra. 
os períodos de férias permitiam-lhe desenvolver sólidos contactos com a vida rural e popular. Depois de estudos universitários, que soube alargar e consolidar por iniciativas próprias, Orlando Ribeiro foi completar a sua formação em Paris. Era, então, o mais activo centro mundial de investigação geográfica. Durante a Guerra e nos anos imediatos, o pacífico Portugal tornou-se palco de intensa investigação científica pluridisciplinar, ainda beneficiada por diversa colaboração internacional. De modo que, nos anos 50, o Centro de Estudos Geográficos já se tinha tornado num foco de investigação activo e produtivo.

João Carlos Garcia evocou, a seguir, a obra de juventude do ainda aprendiz de geógrafo, que começou muito cedo a publicar. Uma versão desenvolvida do seu testemunho, baseada na análise destes textos e enriquecida por documentação recentemente encontrada, será por ele publicada em breve.

Para o segundo colóquio tinha sido escolhido o tema da influência de Orlando Ribeiro sobre a evolução do ensino da Geografia no ensino secundário. Sérgio Claudino soube reunir, para isso, um leque de antigos alunos e discípulos, mais ou menos directos, que vieram testemunhar os modos variados de receber essa influência. $\mathrm{O}$ que ressaltou mais dos depoimentos foi a diversidade de formas de contacto, quer com o seu ensino, quer com a sua obra. Uma verdadeira análise das razões que atrasaram e limitaram bastante, na realidade, a eficácia da influência de Orlando Ribeiro sobre o ensino da Geografia em Portugal, é um assunto importante mas complexo, que Sérgio Claudino continua estudando e pensa poder apresentar em breve, com maior desenvolvimento. Maria Helena Gualberto, Maria Helena Magro e Júlia Galego foram directamente alunas de Orlando Ribeiro. Esta última tornou-se também, mais tarde, sua eficiente colaboradora. Pelo contrário, Arinda Rodrigues, Énio Semedo e o historiador Adérito Tavares só receberam a sua influência através das obras publicadas.

O terceiro colóquio foi concebido por Ilídio do Amaral, "devotado colaborador e amigo, desde meu aluno a meu Reitor", como dirá Orlando Ribeiro em 1981. Esta prolongada e estreita colaboração permitiu-lhe apresentar um retrato intimista e sensível, de feição humanista, de quem foi seu professor e colega. Numa primeira parte, ele evocou as suas constantes preocupações epistemológicas e o seu largo conceito da ciência, baseado num complexo leque de preocupações naturalistas e humanas. A seguir, Ilídio do Amaral alargou-se gostosamente, evocando as tendências musicais e poéticas do geógrafo, e a preocupação que ele sempre teve de partilhar esta "mercadoria espiritual" com os jovens que o rodeavam, devolvendo-lhes assim a generosa iniciação recebida na juventude. Mostrou quanto esta larga, diversificada e sólida substrutura cultural alimentou e enriqueceu os seus temas de investigação, mesmo nas formas especializadas e, aparentemente, técnicas de alguns deles.

O quarto colóquio, proferido por José Veiga Simão, teve o enorme interesse de revelar um aspecto ainda pouco conhecido da personalidade de Orlando Ribeiro. Ele foi sempre, com efeito, um cidadão ansioso de ser directamente útil ao seu país, não através de participação directa na vida política, que sempre o aborreceu, mas como conselheiro respeitado e ouvido por responsáveis gover- 
namentais conscientes e eficazes. Mas tal feliz conjunção só se realizou uma vez e durante apenas um curso lapso de tempo, por ter sido brutalmente interrompido pela Revolução de Abril de 1974. Ela resultou do encontro entre duas fortes personalidades que, para além das suas diferenças, souberam unir vontade e esforços para levar Portugal no caminho da renovação, através da reforma do seu ensino, com exigente procura de excelência. As múltiplas peripécias, pessoais ou nacionais, que seguiram este curto, prometedor mas parcialmente fracassado episódio, nunca fizeram esmorecer nos dois amigos a estima recíproca e o perdurável reconhecimento.

No quinto colóquio, Jorge Gaspar escolheu evocar o que Orlando Ribeiro lhe terá ensinado sobre Lisboa e arredores, a terra natal de ambos. Ele evocou com gosto as visitas pedestres que Mestre Orlando dirigia amiúde e comentava, quer para amigos escolhidos, quer para largos grupos universitários ou internacionais (fig. 3). Discípulo ocasionalmente rebelde, por ter sido sempre cioso em não abdicar de uma ânsia de criação pessoal inovadora, Jorge Gaspar foi, ao mesmo tempo, um amigo próximo e dedicado, capaz tanto de partilhar momentos de simples alegria como de sossegar fundas angústias. Ele foi também, na altura própria, o indispensável renovador de um Centro de Estudos que tinha crescido tanto que a sua gestão exigia uma remodelação profunda.

Era mais do que legítimo que o último colóquio ficasse a cargo de António Ribeiro, o filho mais velho do homenageado, que o pai teve o gosto de ver

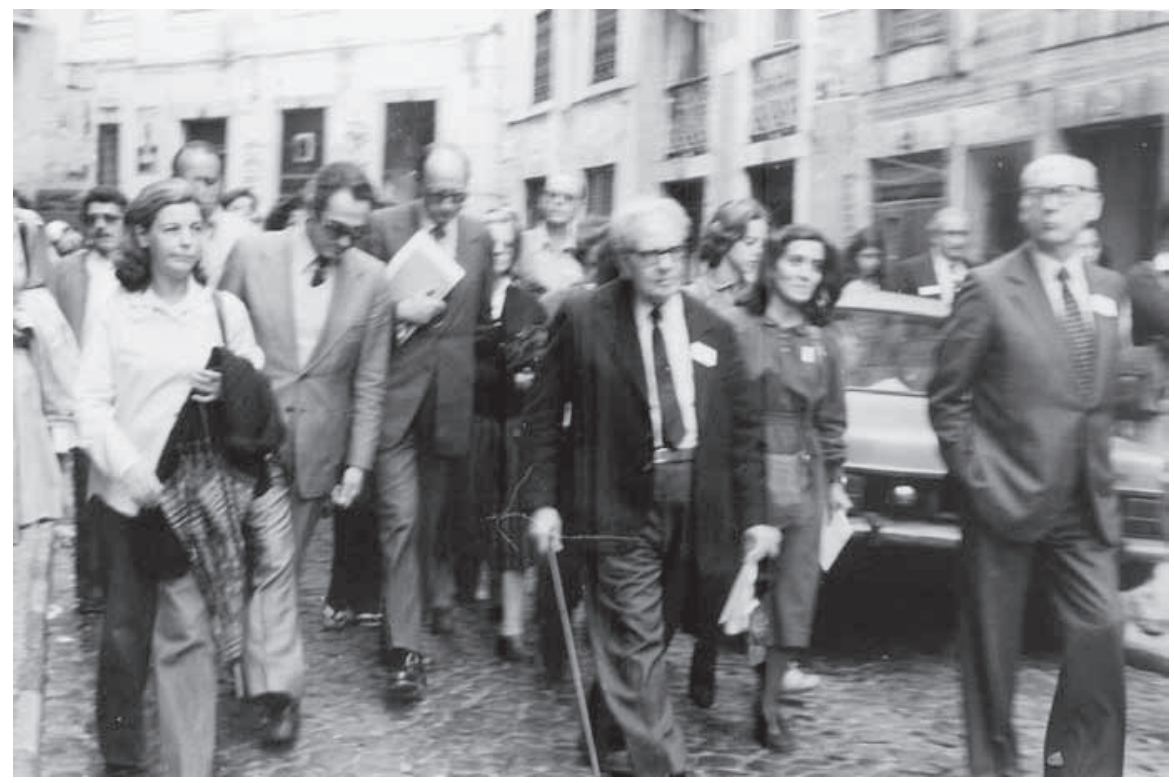

Fig. 3 - Visita ao bairro de Alfama, Lisboa, com os participantes do II Colóquio Ibérico de Geografia, em Outubro de 1980.

Fig. 3 - Visit to the Alfama city district during the 2nd Iberian Geography Conference in 1980. 
chegar ao mais alto nível da criação científica, da irradiação metodológica e da transmissão do saber. "Este menino, a quem ensinei os rudimentos da observação de campo como uma criança aprende uma língua estrangeira", como escrevia Orlando Ribeiro em 1983, soube seguir caminho próprio. "Onde ele procura a exactidão da expressão matemática, busco eu o rigor na condução do raciocínio", segundo ponderou o pai. Trabalhando noutra escala de espaço e de tempo, António Ribeiro quis retomar a lendária formulação do seu pai, para mostrar que a Ibéria é, ao mesmo tempo, mediterrânea e atlântica, não apenas no âmbito dos poucos milénios da história humana, mas também no mais amplo tempo geológico e à medida do planeta.

\section{LA BIBLIOTHÈQUE MUNICIPALE ORLANDO RIBEIRO ET L'HOMMAGE À SON TITULAIRE}

En décembre 2003 fut inaugurée à Lisbonne une toute nouvelle Bibliothèque Municipale, dans le quartier périphérique de Telheiras, alors en plein développement. Agréablement située près du centre mais dans la tranquille partie récupérée du vieux noyau villageois, cette Bibliothèque occupe les bâtiments d'une ancienne résidence à finalité agricole, complétés par des installations nouvelles.

Ce tranquille lieu d'étude et de loisir connut immédiatement un franc succès, qu'aurait beaucoup apprécié son dédicataire Orlando Ribeiro, lui qui, dès l'enfance, avait tant fréquenté les bibliothèques publiques qui lui étaient accessibles. Celle-ci fut conçue pour aider aussi bien les enfants que les adultes à effectuer des Voyages à travers l'information et le savoir et l'idée de lui donner le nom du grand géographe est due tant à son disciple Jorge Gaspar qu'à l'avocat João Soares, alors maire de Lisbonne.

Un ample programme d'hommage fut alors conçu, comportant deux expositions successives et tout un cycle de colloques, retraçant les multiples facettes de l'action culturelle développée par Orlando Ribeiro au long de sa vie. Si le projet initial dut être quelque peu réduit, en raison même du succès croissant et durable des activités proposées aux gens du quartier par la Bibliothèque, il fut cependant possible de réaliser six colloques, dont la substance est reproduite ci-après, dans l'ordre même où ils furent réalisés.

Le premier colloque présenta les années de jeunesse et de formation d'Orlando Ribeiro. Suzanne Daveau illustra photographiquement ses appartenances de jeunesse: la campagne et Viseu, tradition provinciale transmise par ses grands parents maternels; Lisbonne, et surtout le quartier universitaire de São Mamede, où son père était droguiste et où il fit toutes ses études. Les quatre années passées à Paris (1937-40) consolidèrent sa formation et lui assurèrent de nombreux contacts internationaux. Les années de guerre furent pour lui, comme pour bien d'autres jeunes chercheurs portugais, le temps de la découverte de leur propre pays. L'organisation do Congrès Géographique International de Lisbonne, en 1949, consolida la stature internationale du Centre d'Études Géographiques, créé par lui en 1943. L'étude, alors présentée par João Carlos Garcia, des écrits de jeunesse du géographe, est en voie d'approfondissement et elle sera bientôt publiée sous une forme plus achevée.

Le second colloque fut centré sur l'influence que la rénovation de la Géographie portugaise par Orlando Ribeiro a pu avoir sur l'évolution de l'enseignement secondaire 
de cette discipline au Portugal. Sérgio Claudino avait réuni pour cela les témoignages de six anciens élèves ou disciples plus ou moins directs du géographe. Chacun raconta la façon dont il reçut son influence, directement ou à travers la lecture de son œuvre. Une de ces élèves, Júlia Galego, deviendra par la suite une précieuse collaboratrice du vieux maître. L'étude des divers facteurs qui limitèrent, en réalité, l'efficacité didactique de son influence est actuellement en cours d'approfondissement par Sérgio Claudino.

Le troisième colloque fut conçu par Ilídio do Amaral, un des collaborateurs les plus proches et les plus durables d'Orlando Ribeiro, puisqu'il fut aussi bien son élève que son Recteur. C'est un portrait intime et approfondi des facettes les plus personnelles de l'identité spirituelle d'Orlando Ribeiro. Dans une première partie, est commentée sa conception de la science, un large éventail allant de la nature à l'homme, et sa constante préoccupation épistémologique. Ensuite sont évoquées la profondeur et l'ampleur de ses goûts musicaux et littéraires, sa pratique personnelle épisodique de la création poétique et son souci constant de faire partager par tout son entourage l'initiation culturelle reçue dans sa jeunesse. Est souligné aussi le fait que cette large culture humaniste a constitué le solide substratum de toutes ses recherches, même les plus techniques ou particulières.

Le quatrième colloque présente un aspect bien différent de la personnalité d'Orlando Ribeiro, celui du citoyen qui aurait tant souhaité servir de conseiller éclairé et écouté par les responsables gouvernementaux de son pays. En fait, ce ne fut que de façon très épisodique, et tôt interrompue, qu'il put avoir cette influence, en 1970-74, grâce à sa rencontre avec l'actif ministre de l'éducation, José Veiga Simão. Ce fut alors la collaboration de deux fortes personnalités qui, au delà de leurs différences, communiaient dans un désir énergique de rénovation du Portugal par la réforme de son enseignement. Le témoignage du grand homme d'action sur son ancien ami et collaborateur, montre quelle fut la profondeur de leur entente et la solidité de leur estime réciproque.

Lors du cinquième colloque, le géographe Jorge Gaspar entendit rappeler les enseignements reçus d'Orlando Ribeiro relativement à la région de Lisbonne, leur ville natale à tous deux. Il évoqua les visites pédestres si souvent dirigées par le Professeur, refaisant photographiquement, pour les actuels auditeurs, les trajets habituels de ces excusions pédagogiques. Surgirent ainsi quelques permanences des paysages urbains et suburbains parcourus, mais surtout les profondes mutations qui les affectèrent au cours des dernières décennies. Disciple particulièrement actif et créatif d'Orlando Ribeiro, Jorge Gaspar sera, durant une longue période, son successeur à la tête du Centre d'Études Géographiques de Lisbonne.

Le cycle des colloques s'acheva enfin sur l'hommage rendu au grand géographe par son propre fils, le géologue António Ribeiro. S'inspirant du titre du livre le plus connu de son père, il exposa ses propres conceptions sur le passé et le futur géologique probable de la Péninsule Ibérique, en montrant quelle est la position unique de L'Ibérie, entre la Méditerranée et l'Atlantique. Une façon scientifique et poétique, à la fois, de replacer les hommes et leur destin dans une échelle qui les dépasse.

\section{THE ORLANDO RIBEIRO CITY LIBRARY AND THE TRIBUTE TO ITS TITULAR}

In December 2003, a new City Library opened in Lisbon in the rapidly growing peripheral quarter of Telheiras. Located in a central but pleasant old part of that quarter 
the Library occupies what once was a farming mansion, as well as several new buildings.

The local residents very quickly came to appreciate this pleasant new place for study and leisure. Orlando Ribeiro himself would have liked it too, in the same way as he once liked the old Town Libraries of his youth. This Library was designed with the aim of helping both children and grown-ups to travel through the world of information and knowledge. The choice of the geographer Orlando Ribeiro as its titular was made by his former pupil Jorge Gaspar and by João Soares, then Mayor of Lisbon.

The large number of activities that made up the tribute program included two exhibitions and a series of conferences on the various aspects of Orlando Ribeiro's longlasting and diversified cultural influence. Even though the original project could not be carried out in full, it was nevertheless possible to organize six conferences. Those six texts, carefully revised by the authors, are reproduced in this volume.

The first conference introduced the childhood and training years of Orlando Ribeiro. Suzanne Daveau showed a series of photographs that illustrated his twin origins: the Beira region, from which his parents originated, and the Lisbon quarter where his father owned a drugstore and where he lived throughout his initial school years. The four years that he spent at the Paris Geographical Institute then provided him with a remarkable scholarly training and enabled him to forge his first international relationships. Then, after the Second World War, which constituted a time of intensive scientific investigation in Portugal, the hosting of the 16th International Geographical Congress by the Centro de Estudos Geográficos in 1949 proved that the young Lisbon centre was already a scientific force to be reckoned with. The study of Orlando Ribeiro's early papers by João Carlos Garcia will be published in an upcoming paper.

The second conference aimed at showing the influence of the revival of Portuguese geography due to Orlando Ribeiro upon the teaching of this discipline at the grammar school level. Sérgio Claudino asked six teachers of these schools to recall the various ways in which they felt that influence: through direct teaching, by reading his books and as of pedagogical trips both to the countryside and in urban areas. Júlia Galego, one of these teachers and disciples of Orlando Ribeiro, would eventually become a close collaborator of the ageing scientist. Why his didactic influence in the end turned out to be smaller than might have been expected is one of Sérgio Claudino's current research interests.

The third conference was a friendly testimony by a long-lasting collaborator, Ilídio do Amaral. He spoke of Orlando Ribeiro's epistemological interests and scientific ideas. Special emphasis was given to Orlando Ribeiro's musical and literary tastes, namely by recalling his episodic yet beautiful poems, and to how much he enjoyed sharing with the young people that surrounded him the cultural heritage that had been passed on to himself during his own youth.

The fourth conference highlighted a very different side of Orlando Ribeiro's personality. He was always a patriot and a citizen eager to assist the country's political leaders. However, this desire was only rendered concrete during the short period between 1970 and 1974, when he met and collaborated with the courageous minister Veiga Simão. They both fought hard to renovate their country through the renewal of education. The friendly testimony presented by the minister showed how deep their reciprocal appreciation was.

In the fifth conference, Jorge Gaspar celebrated the way in which Orlando Ribeiro shared his knowledge of Lisbon, the city were both were born. He recalled the numer- 
ous pedestrian trips led by Orlando Ribeiro throughout the city and its suburbs, and used a series of photographs to highlight the drastic changes that the city's landscape has undergone over the past half century. One of Orlando Ribeiro's most creative disciples, Jorge Gaspar succeeded him as Director of CEG for many years.

The responsibility for the sixth and final conference fell upon one of Orlando Ribeiro's sons, the geologist António Ribeiro. He referred to the title of his father's most famous book, Portugal, o Mediterrâneo e o Atlântico, in order to illustrate how Ibéria, this tiny continent, is truly both Mediterranean and Atlantic in cha-racter, not only from the point of view of human history, but also in the broadest possible geological sense. 